\title{
Skin malignancy initially misdiagnosed as a benign epidermal cyst
}

\author{
Chan Min Chung ${ }^{1}$, \\ Sung Jae Wee, \\ Hyoseob Lim ${ }^{1}$, \\ Sang Hun Cho', \\ Jong Wook Lee ${ }^{2}$ \\ ${ }^{1}$ Department of Plastic and \\ Reconstructive Surgery, Hallym \\ University Sacred Heart Hospital, \\ Hallym University College of Medicine, \\ Anyang; ${ }^{2}$ Department of Plastic and \\ Reconstructive Surgery, Hallym \\ University Hangang Sacred Heart \\ Hospital, Seoul, Korea
}

\begin{abstract}
Skin cancer, which often occurs as a result of skin exposure to ultraviolet light radiation, usually presents with characteristic abnormal features, such as ulcerative lesions, irregular morphology, bleeding, and excessive growth. Therefore, skin cancer rarely resembles a benign tumor on visual inspection. Nonetheless, squamous cell carcinoma and basal cell carcinoma with nodular or polypoid features can have a similar appearance to that of benign tumors, meaning that they are sometimes misdiagnosed as benign. As benign and malignant tumors have some overlapping features, clinicians sometimes use additional imaging techniques such as ultrasonography to improve the accuracy of the diagnosis because even a malignant tumor that externally resembles a benign tumor generally has internal morphological features characteristic of malignancy, such as invasion and irregular borders. However, these imaging tools also have limitations, and punch or excisional biopsy can be needed if malignancy cannot be completely ruled out. Herein, we report a case of skin malignancy initially misdiagnosed as a benign epidermal cyst based on external visual inspection and ultrasonography.
\end{abstract}

Keywords: Carcinoma, basal cell / Carcinoma, squamous cell / Epidermal cyst / Ultrasonography

\section{INTRODUCTION}

Skin malignancy usually occurs as a result of chronic damage from sunlight [1]. Squamous cell carcinoma (SCC) or basal cell carcinoma (BCC) may show pigmented lesions and various morphological features, including ulcerative or polypoid morphologies. SCC or BCC with nodular morphologies can be mistaken for other benign nodular tumors. In an analysis of skin tumors, Lin et al. [2] suggested that benign and malignant nodular tumors had some overlapping features. Therefore, imaging techniques such as ultrasonography (US), computed tomography $(\mathrm{CT})$, and magnetic resonance imaging are used to improve diagnostic accuracy for lesions suspected to be skin

\footnotetext{
Correspondence: Hyoseob Lim

Department of Plastic and Reconstructive Surgery, Hallym University Sacred Heart Hospital, Hallym University College of Medicine, 22 Gwanpyeong-ro

170beon-gil, Dongan-gu, Anyang 14068, Korea

E-mail: hyoseob.lim@gmail.com

Received December 8, 2019 / Revised March 18, 2020 / Accepted March 26, 2020
}

cancer. This paper reports a case of skin malignancy in the left periauricular region that was initially misdiagnosed as a benign epidermal cyst based on external visual inspection and US.

\section{CASE REPORT}

An 81-year-old man with three masses in his left periauricular region was referred to our department. The patient reported that he originally had a nevus in the region and underwent a nevus removal procedure at a dermatology clinic 2 years ago.

There were two posterior masses (upper, $2.6 \times 2.0 \mathrm{~cm}$; lower, $1.7 \times 1.4 \mathrm{~cm}$ ) and one anterior mass. The two posterior masses were erythematous dome-shaped nodules connected to each other. Meanwhile, the anterior mass $(1.2 \times 1.2 \mathrm{~cm})$ was smaller than the other two masses and showed a small ulcerative opening covered by a crust (Fig. 1). To assess the lesions more accurately, we performed US, which showed well-marginated, hyperechoic masses with tiny internal echogenic spots. In addi- 


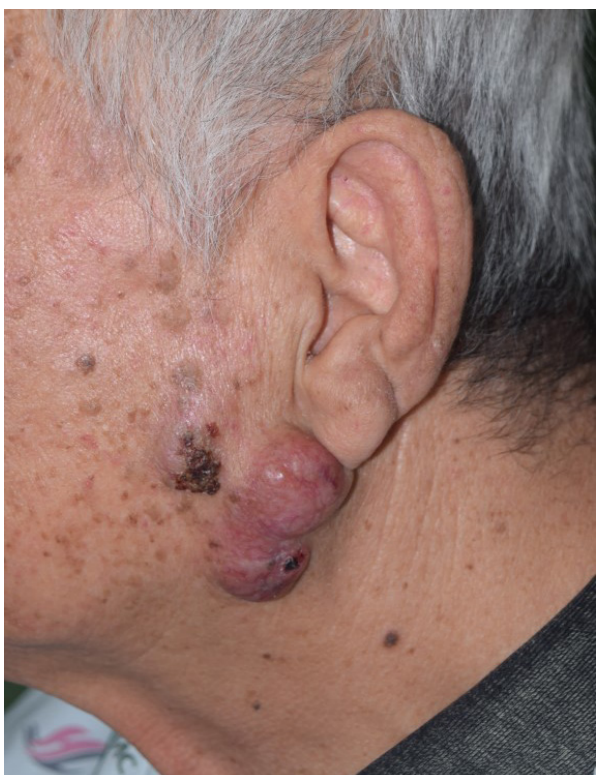

Fig. 1. An 81-year-old man with three masses in his left periauricular region. The two posterior masses (upper mass, $2.6 \times 2.0 \mathrm{~cm}$; lower mass, $1.7 \times 1.4 \mathrm{~cm})$, and the anterior mass $(1.2 \times 1.2 \mathrm{~cm})$ are shown. tion, there was peripheral hyperechogenicity of fat tissue surrounding the masses with associated posterior enhancement. These US findings led to the strong suspicion that the three lesions were benign tumors, such as epidermal cysts with possible associated inflammation (Fig. 2).

An excisional biopsy was performed under local anesthesia. A lazy S-shaped incision was made to expose all the three masses. Intraoperatively, we found that the three masses were composed of lumps of necrotic tissue, instead of showing the expected characteristics of a cystic mass. A histopathological examination showed atypical keratinocytes with hyperchromatic nuclei growing into the dermis suggesting that the anterior mass could be SCC (Fig. 3A). The posterior masses showed basaloid cell proliferation with peripheral palisading and cleft between tumor and stroma, raising the possibility that they were connected BCCs (Fig. 3B). Although an additional wider local excision was strongly recommended to the patient and his guardian on the basis of the histopathological findings, they rejected any additional surgery owing to the patient's overall
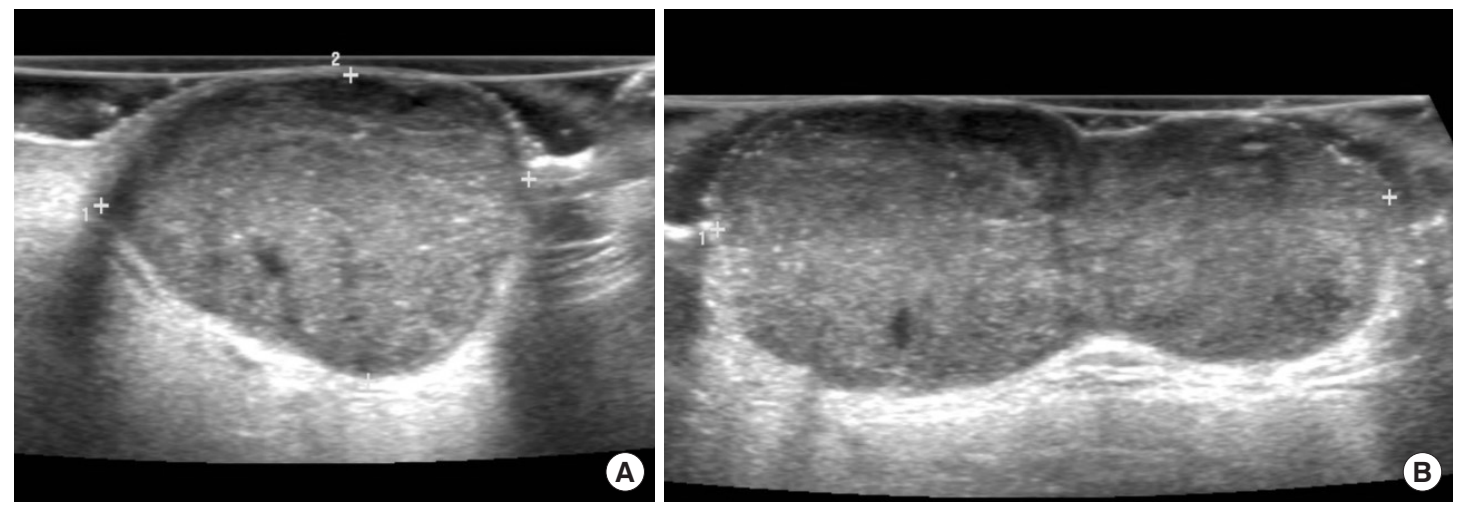

Fig. 2. Visualization of the masses on ultrasonography. Ultrasonography showed (A) the anterior mass and (B) the posterior masses, which were connected with each other.
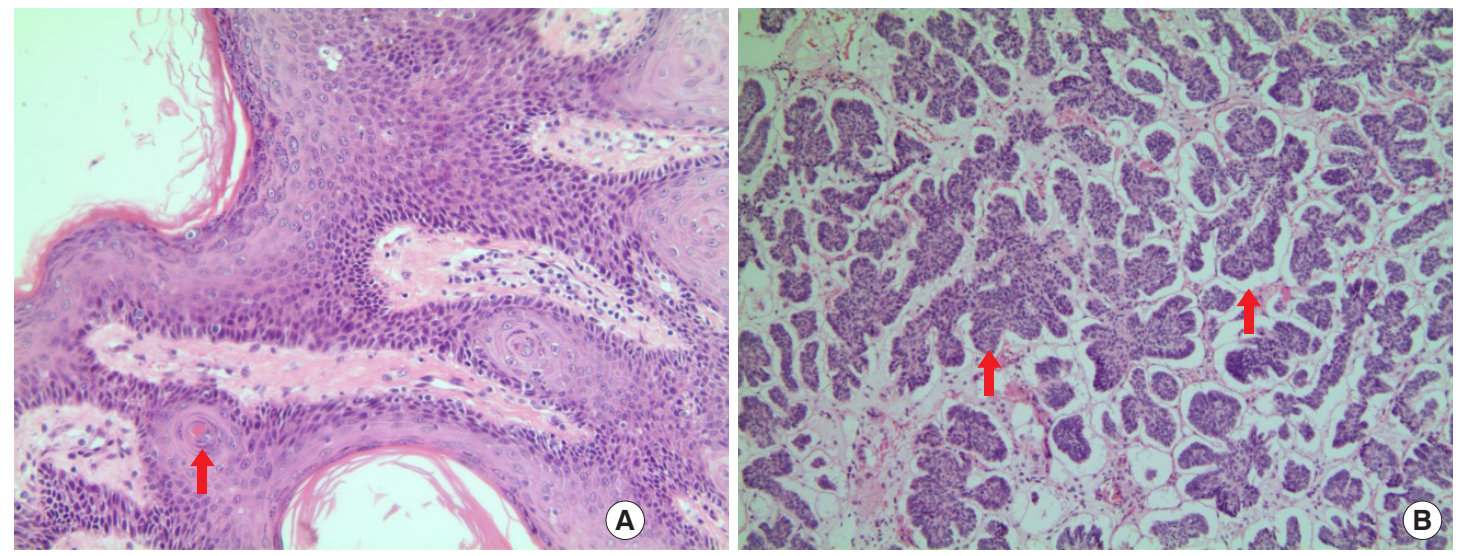

Fig. 3. Histopathological images showing squamous cell carcinoma and basal cell carcinoma. (A) Atypical keratinocytes with hyperchromatic nuclei (arrow, H\&E, $\times 200$ ) and (B) basaloid cell proliferation with peripheral palisades and a cleft between the tumor and stroma (arrows, $\mathrm{H} \& \mathrm{E}, \times 100)$. 


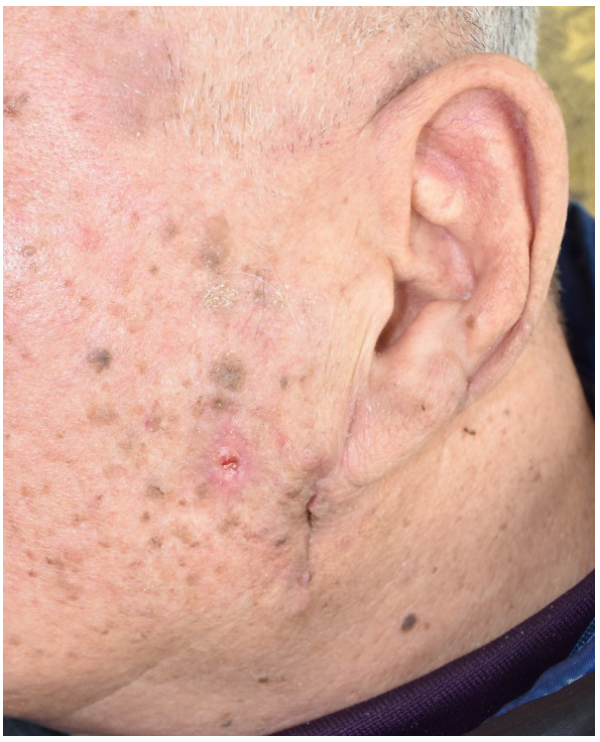

Fig. 4. Postoperative photograph 3 weeks after surgery. Two small wounds are shown where the masses were located.

health and the potential risk from an additional surgical procedure. The surgery site was completely epithelialized except for two small openings (Fig. 4). One month after surgery, neck, chest, and abdominopelvic CT was taken for a cancer metastasis work-up. The CT findings showed several skin thickenings with enhancement in the left periauricular area, confirming the presence of residual tumor tissue (Fig. 5). However, we found no regional lymph node metastasis or distant metastasis on CT, and the tumor was confirmed to be pT2N0M0 (i.e., stage II), according to the American Joint Committee on Cancer staging manual, eighth edition [3].

Over a 2-year period, the patient and his guardian were informed of conservative treatment methods such as avoiding additional ultraviolet exposure and keeping the affected area clean. Although the discharge persisted for 2 years, we observed no findings that suggested malignant growth, such as increased lesion size, additional ulceration, or a polypoid lesion.

\section{DISCUSSION}

The epidemiological characteristics of skin malignancies, especially BCC and SCC, have been thoroughly analyzed. According to previous studies, a leading risk factor of skin malignancy is exposure to ultraviolet light radiation [4]. Exposure to ionizing radiation, exposure to arsenic-contaminated materials, a weakened immune system, genetic problems, and smoking and drinking habits have been identified as additional risk factors of skin malignancies [5]. However, laser therapy or intense pulsed light therapy, which are procedures mostly used in dermatolo-

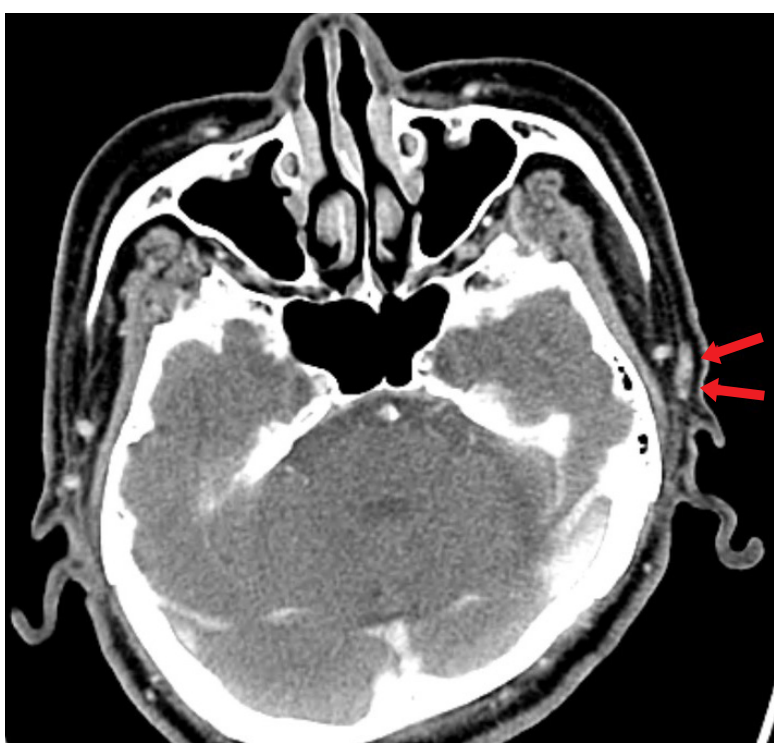

Fig. 5. Postoperative computed tomography scan showing the residual tumor. Several areas of skin thickening with enhancement in the left periauricular area (arrows) confirmed the presence of residual tumor tissue.

gy, have not only prevented the incidence of skin cancer from increasing for the last two decades, but have also shown a therapeutic effect on skin cancer [6]. Therefore, in the present case, we cannot completely exclude the possibility that the previous dermatological procedure performed to remove the patient's nevus may have coincided with the incidence of the skin malignancy, which had already begun to grow.

Several studies have also explored associations between benign and malignant skin tumors. Veenstra et al. [7] retrospectively analyzed 1,904 epidermal cyst cases and confirmed that only three developed into malignant tumors. A proposed hypothesis for the malignant transformation of a benign tumoralthough it is a rare event-is that "chronic stimulation" around the mass caused by material inside the mass may lead to chronic inflammation, resulting in eventual progression into a malignant tumor. Although excision is not necessary for benign tumors, such as epidermal cysts or pilomatricomas, as long as they remain small and cause no problems, a histological examination of the tumor is necessary if it shows clinically or radiologically malignant features such as rapid growth or irregular morphology [8]. Meanwhile, in some cases, additional benign tumors were found in skin malignancies. Reinders et al. [9] reported a case in which an epidermal cyst newly developed in a preexisting BCC, and a case where an epidermal cyst was found in a melanoma was also reported [10].

US findings are very helpful in the diagnosis of soft tissue tumors. On US, an unruptured epidermal cyst presents as a wellcircumscribed mass, with a heterogeneously and mildly echo- 
genic appearance. A ruptured epidermal cyst is usually seen as an irregularly marginated hypoechoic lesion, and an infected epidermal cyst shows increased blood flow in and at the periphery of the mass on Doppler US [11]. In contrast, tumors have pleomorphic appearances, as they may present as lobulated, asymmetric, irregular, or bulging lesions with hyperechoic spots. In addition, tumors characteristically show increased vascularity into the lesion on Doppler US [12]. However, the diagnosis of soft tissue tumors on the basis of US findings alone has limitation. Some malignant tumors can mimic benign tumors, as seen in our case, where the US features (well-circumscribed margin, heterogeneity, and posterior enhancement) were suggestive of a benign tumor. In light of these US findings, simple excision was performed, but the biopsy confirmed the diagnosis as a malignant tumor.

This case report has some limitations. First, no further pathological findings were obtained, as an additional wide local excision was not performed because the patient and his guardian refused. Had a wide local excision been performed, we might have obtained additional information about how the tumor developed in this case. Second, we could not precisely determine the dermatological procedure performed 2 years before the patient underwent the present excision, so we were not able to explore its possible association with his skin cancer.

In the present case, it was unclear whether a benign tumor developed first and then turned malignant, or a malignant tumor developed first and simply showed the morphology of a benign tumor. However, physicians must suspect skin malignancy in the presence of any indication that suggests a malignant lesion, even if a lesion presents features similar to those of a benign tumor on visual inspection and has radiological findings implying benignity.

\section{NOTES}

\section{Conflict of interest}

No potential conflict of interest relevant to this article was reported.

\section{Ethical approval}

The study was approved by the Institutional Review Board of Hallym Sacred Heart Hospital (IRB No. 2019-10-001) and performed in accordance with the principles of the Declaration of Helsinki. Written informed consent was obtained.

\section{Patient consent}

The patient provided written informed consent for the publication and the use of his images.

\section{ORCID}

Chan Min Chung https://orcid.org/0000-0002-2755-9440

Sung Jae Wee https://orcid.org/0000-0002-2397-7450

Hyoseob Lim https://orcid.org/0000-0002-6251-8281

Sang Hun Cho https://orcid.org/0000-0001-5906-9828

Jong Wook Lee https://orcid.org/0000-0002-3244-949X

\section{REFERENCES}

1. Narayanan DL, Saladi RN, Fox JL. Ultraviolet radiation and skin cancer. Int J Dermatol 2010;49:978-86.

2. Lin MJ, Pan Y, Jalilian C, Kelly JW. Dermoscopic characteristics of nodular squamous cell carcinoma and keratoacanthoma. Dermatol Pract Concept 2014;4:9-15.

3. Amin MB, Edge SB, Greene FL, Byrd DR, Brookland RK, Washington MK, et al. AJCC Cancer Staging Manual. 8th ed. New York: Springer; 2017.

4. Marzuka AG, Book SE. Basal cell carcinoma: pathogenesis, epidemiology, clinical features, diagnosis, histopathology, and management. Yale J Biol Med 2015;88:167-79.

5. Marur S, Forastiere AA. Head and neck squamous cell carcinoma: update on epidemiology, diagnosis, and treatment. Mayo Clin Proc 2016;91:386-96.

6. Ash C, Town G, Whittall R, Tooze L, Phillips J. Lasers and intense pulsed light (IPL) association with cancerous lesions. Lasers Med Sci 2017;32:1927-33.

7. Veenstra JJ, Choudhry S, Krajenta RJ, Eide MJ. Squamous cell carcinoma originating from cutaneous cysts: The Henry Ford Experience and review of the literature. J Dermatolog Treat 2016;27:95-8.

8. Bajoghli A, Agarwal S, Goldberg L, Mirzabeigi M. Melanoma arising from an epidermal inclusion cyst. J Am Acad Dermatol 2013;68:e6-7.

9. Reinders MG, Brinkhuizen T, Soetekouw PM, Kelleners-Smeets NW, Hamid MA, Mosterd K. Epidermal cyst formation and hyperkeratosis in a patient treated with vismodegib for locally advanced Basal cell carcinoma. Acta Derm Venereol 2015;95: 618-9.

10. Swygert KE, Parrish CA, Cashman RE, Lin R, Cockerell CJ. Melanoma in situ involving an epidermal inclusion (infundibular) cyst. Am J Dermatopathol 2007;29:564-5.

11. Chung HW, Cho KH. Ultrasonography of soft tissue "oops lesions." Ultrasonography 2015;34:217-25.

12. Wortsman X. Sonography of facial cutaneous basal cell carcinoma: a first-line imaging technique. J Ultrasound Med 2013;32: $567-72$. 